\title{
A visão das crianças sobre a felicidade
}

\author{
Claudia Hofheinz Giacomoni \\ Universidade Federal do Rio Grande do Sul - RS \\ Luciana Karine de Souza \\ Universidade Federal de Minas Gerais - MG \\ Claudio Simon Hutz \\ Universidade Federal do Rio Grande do Sul - RS
}

\begin{abstract}
Resumo
O objetivo deste trabalho foi compreender as concepções da criança sobre a experiência de ser feliz e de como seja uma pessoa assim, conforme a faixa etária e o nível socioeconômico. Participaram 200 crianças (52,5\% meninas) de cinco a doze anos, de escolas públicas e privadas. As crianças foram entrevistadas individualmente sobre o que é ser feliz e como é uma pessoa feliz. Sobre o que é ser feliz, as crianças trouxeram mais conteúdos sobre sentimentos positivos e self positivo. As crianças de escolas privadas mencionaram mais o lazer, e as de escola pública destacaram a satisfação de necessidades básicas e desejos. Sobre como é uma pessoa feliz, mais da metade da amostra apontou características subjetivas positivas, com os meninos citando mais esses conteúdos. Os resultados são discutidos com base na literatura disponível, com implicações na avaliação do bem-estar subjetivo e da satisfação de vida na infância.
\end{abstract}

Palavras-chave: Crianças, felicidade, bem-estar da criança.

\section{Children's view on happiness}

\begin{abstract}
The aim of this study was to comprehend children's description of the experience of being happy as well as the appearance of a happy person. Age range and socioeconomic level were analyzed. Two hundred children, aged from 5 to 12 years-old, from public and private schools located in Porto Alegre ,Brazil, participated in the study. Children were interviewed individually about what it means to be happy and how a happy person looks like. As for the experience of being happy, children mentioned more positive feelings and a sense of positive self. Private school children cited more leisure activities, whilst public school children referred more to the need to satisfy basic needs and personal desires. More than half of the children interviewed described a happy person through positive subjective characteristics, mainly answers given by boys. Results are discussed based on the available literature, with implications to the assessment of subjective well-being and life satisfaction in childhood.
\end{abstract}

Keywords: Children, happiness, child welfare.

\section{La comprensión de los niños sobre la felicidad}

\section{Resumen}

El objetivo de este estudio fue comprender las concepciones de niños sobre la experiencia de ser feliz y de cómo sería una persona así, de acuerdo a la franja etaria y nivel socioeconómico. Participaron 200 niños $(52,5 \%$ niñas) de cinco a doce años, de escuelas públicas y privadas. Los niños fueron entrevistados individualmente sobre lo que es ser feliz y cómo es una persona feliz. Acerca de lo que es ser feliz, los niños mencionaron más contenidos sobre sentimientos positivos y self positivo. Los niños de escuelas privadas mencionaron más la diversión en tiempo libre y los de escuela pública resaltaron la satisfacción de necesidades básicas y deseos. Sobre cómo es una persona feliz, más de la mitad de la muestra mostró características subjetivas positivas, con los niños citando más esos contenidos. Se discuten los resultados con base en la literatura disponible, con implicaciones en la evaluación del bienestar subjetivo y de la satisfacción de vida en la infancia.

Palabras Clave: Niños, felicidad, bienestar del niño. 


\section{Introdução}

A pesquisa psicológica de experiências, traços e estados positivos é escassa no Brasil, especialmente estudos sobre felicidade. O movimento da Psicologia Positiva ainda é tímido no país, embora núcleos de pesquisa e laboratórios estejam já empreendendo esforços nesse sentido (Dell'Aglio, Koller, \& Yunes, 2006; Hutz, no prelo; Seligman \& Csikszentmihalyi, 2000). Ainda assim, há trabalhos dedicados a temas positivos, como as pesquisas de La Taille (2006) sobre generosidade infantil e Freitas, Silveira e Pietá (2009) sobre gratidão em crianças.

Os trabalhos de Huebner e Gilman (2002) sobre a satisfação de vida infantil têm mostrado cinco domínios específicos nessa abordagem: família, amizade, escola, ambiente e self. Nesses moldes, Giacomoni e Hutz (2008) construíram uma escala para avaliar a satisfação de vida em crianças, encontrando seis fatores consistentes no tratamento psicométrico do construto estudado: self, self comparado, não-violência, família, amizade e escola. No caso do bem-estar subjetivo infantil, carecem dados sobre como as crianças brasileiras vivenciam a experiência de felicidade, sob variadas condições (por exemplo, nível socioeconômico). Conhecer como a criança constrói a idéia de ser feliz e de como é uma pessoa feliz está relacionado à forma como a criança percebe a si e a outrem.

Há pesquisas nacionais com resultados interessantes sobre alguns dos domínios da satisfação de vida na infância. Levy e Jonathan (2010) analisaram a redação de 52 crianças fluminenses sobre "minha família", estudantes de escolas públicas e privadas, com idade média de 10 anos, $63 \%$ meninas. Nesses textos, as crianças da escola privada foram as que mais citaram lazer em família $(p<0,02)$, ao passo que as de escola pública mencionaram mais conflitos, embora a diferença não tenha sido significativa para o outro grupo. O que a pesquisa de Levy e Jonathan (2010) sugere é que a criança vê, na família, experiências positivas como a de lazer, o que muito provavelmente inclui atividades divertidas para a criança.

Quanto à amizade em crianças, Garcia e Pereira (2008) conduziram um estudo com 40 crianças de 7 a 10 anos de idade da cidade de Vitória (ES), através do qual investigaram vários aspectos destes relacionamentos. Dentre os resultados interessantes, percebeu-se que a emoção mais associada à amizade nessas crianças foi a de alegria, e que as qualidades dos amigos incluem ter brincadeiras legais, ser uma pessoa boa, ser honesto, ter bom caráter, ser inteligente, bonito, alegre, sorridente. Portanto, é de se esperar que as crianças incluam a amizade como componente da experiência de uma pessoa feliz.

Sobre o autoconceito em crianças, Cia e Barham (2009) avaliaram 99 crianças de 6 a 9 anos de idade, estudantes de escolas públicas de um município do interior do estado de São Paulo. O autoconceito foi medido por uma escala de 76 itens, em oito subescalas: habilidades físicas, aparência física, relacionamento com os colegas, com os pais (autoconceito não-acadêmico), leitura, matemática, assuntos escolares em geral (autoconceito acadêmico), e autoconceito geral. O autoconceito das crianças da amostra foi considerado satisfatório (escore máximo de 40,0): autoconceito total $=29,5$; autoconceito não-acadêmico $=28,8$; e autoconceito acadêmico $=30,2$. Este último escore vem a confirmar a importância da vida escolar para a criança. Dado seu empenho e tempo dedicado à escola e suas atividades, é importante para ela desempenhar bem nas atividades escolares, o que favorece a imagem de si. Nessa idade especialmente, a criança ainda utiliza bastante os aspectos mais comportamentais em suas autodescrições, como em "sou a melhor desenhista da turma", "sou o melhor em matemática da sala” (Harter, 1999). Assim, com relação à visão da criança sobre a felicidade, é lícito considerar que sua definição inclua experiências positivas com a escola.

Com respeito à não-violência em crianças, Souza, Sperb, McCarthy e Biaggio (2006) investigaram as concepções de paz, de guerra e de violência em 61 crianças com média de idade de 7,6 anos, e 63 pré-adolescentes com idade de 12,7 anos, em média, estudantes de uma escola pública da cidade de Porto Alegre (RS). Metade da amostra definiu paz e violência, e a outra metade definiu guerra e violência. Dos 64 alunos que definiram paz, a maioria (73\%) deles mencionou os conteúdos de felicidade, amor e união, tendo em segundo lugar como mais citada a categoria negação da violência (53\%). Estes resultados sugerem que a ideia de paz em crianças do sul do Brasil está bastante relacionada à felicidade, especialmente em nível interpessoal (união, amor), e à não-violência (em nível social, como a ausência de crimes, de roubos). Assim, pode-se esperar que a visão da criança sobre ser feliz e sobre uma pessoa feliz esteja associada à ausência de violência, de conflitos, e à presença de emoções positivas e de felicidade, principalmente em nível interpessoal.

O presente estudo é parte inédita de uma pesquisa maior sobre bem-estar e qualidade de vida infantil. O objetivo, com este trabalho, foi conhecer o que a criança entende por ser feliz e como a criança imagina que seja uma pessoa feliz. A expectativa é de identificar concepções diferentes para a experiência de ser feliz e de como seja uma pessoa assim, conforme a faixa etária e o nível socioeconômico.

\section{Método}

Participaram da presente pesquisa 200 crianças da cidade de Porto Alegre, com idade média de 8,7 anos (DP = 2,5; cinco faixas etárias de 5 a 12 anos), sendo $52,5 \%$ meninas. Os participantes frequentavam escolas públicas ou privadas. Nesse sentido, buscou-se uma aproximação ao nível socioeconômico, neste estudo abordado através do tipo de escola.

As crianças responderam a uma entrevista semi-estruturada individual, contendo as seguintes questões: 1) O que é ser feliz?, e 2) Como tu achas que uma pessoa feliz é? Os pais/responsáveis assinaram um termo de consentimento, e o estudo foi aprovado pelo comitê de ética em 
pesquisa. As entrevistas foram realizadas na própria escola em sala específica para esse fim.

As entrevistas foram transcritas e submetidas a análise de conteúdo (Bardin, 2011). Três avaliadores independentes criaram categorias de respostas com unidades temáticas semelhantes, e a concordância interjuízes ficou em torno de $80 \%$. Foram efetuados cálculos de frequência e de porcentagem de resposta por categoria, considerando o tipo de escola (pública ou privada), o sexo das crianças, e os grupos etários 5-6 anos, 7-8, 9-10 e 11-12 anos.

Para as comparações entre os grupos de escola privada e pública, foram compostos dois grupos de alunos pareados por faixa etária e série escolar, considerando-se a quantidade de crianças da escola privada $(n=43)$. Foi utilizado o Teste Binomial para Diferenças entre Duas Proporções (Hinkle, Wiersma, \& Jurs, 1988) e o Teste do Qui-Quadrado para Várias Proporções Populacionais (nível de significância de 0,05) (Ayres, Ayres Jr, Ayres, \& Santos, 2000).

\section{Resultados}

Com relação à primeira questão, sobre o que é ser feliz, as respostas analisadas permitiram a emergência de dez categorias excludentes: sentimentos e estados positivos (rir, felicidade, alegria), self positivo e altruísmo (ser legal, repartir, ajudar, amar), lazer (atividades físicas e divertidas, brincar), satisfação de necessidades básicas e desejos (ter casa, comida, ganhar presentes), família (referências à família), amizade (referências aos relacionamentos com os pares), não-violência (não ser ator nem expectador de violências), escola (referências à escola), outras respostas, e não respondeu.

A Tabela 1 apresenta a frequência, porcentagem e o resultado da comparação de sexo nas categorias sobre o que é ser feliz. Não constam na tabela as categorias "outras respostas", com 12 crianças, e "não respondeu" (com oito crianças).

Tabela 1. Frequência e porcentagem de respostas para o que é ser feliz, por sexo.

\begin{tabular}{lcccccccc}
\hline & \multicolumn{2}{c}{ total } & \multicolumn{2}{c}{ Meninas } & \multicolumn{2}{c}{ Meninos } \\
\cline { 2 - 9 } & $n$ & $f(\%)$ & $n$ & $f(\%)$ & $n$ & $f(\%)$ & $Z$ & $p$ \\
\hline Sentimentos & 82 & $106(27,5)$ & 45 & $54(27,8)$ & 37 & $52(27,2)$ & 0,13 & 0,89 \\
Self Positivo & 38 & $57(14,8)$ & 17 & $25(12,9)$ & 21 & $32(16,8)$ & $-1,06$ & 0,28 \\
Lazer & 39 & $53(13,7)$ & 20 & $25(12,9)$ & 19 & $28(14,7)$ & $-0,50$ & 0,61 \\
Neces. básicas/desejos & 41 & $52(13,5)$ & 16 & $24(12,4)$ & 25 & $28(14,7)$ & $-0,65$ & 0,51 \\
Família & 27 & $34(8,8)$ & 17 & $22(11,3)$ & 10 & $12(6,3)$ & 1,74 & 0,08 \\
Amizade & 27 & $28(8,2)$ & 15 & $15(7,7)$ & 12 & $13(6,8)$ & 0,34 & 0,72 \\
Não-Violência & 17 & $20(5,1)$ & 9 & $11(5,7)$ & 8 & $9(4,7)$ & 0,42 & 0,67 \\
Escola & 12 & $14(3,6)$ & 5 & $7(3,6)$ & 7 & $7(3,7)$ & $-0,02$ & 0,97 \\
\hline
\end{tabular}

Tabela 2. Frequência e porcentagem de respostas para o que é ser feliz, por escola.

\begin{tabular}{lcccccc}
\hline & \multicolumn{2}{c}{ Pública } & \multicolumn{2}{c}{ Privada } & \\
\cline { 2 - 7 } & $n$ & $f(\%)$ & $n$ & $f(\%)$ & $Z$ & $p$ \\
\hline Sentimentos & 15 & $20(20,8)$ & 18 & $27(27,8)$ & $-1,13$ & 0,25 \\
Self Positivo & 12 & $17(17,7)$ & 13 & $21(21,6)$ & $-0,68$ & 0,49 \\
Lazer & 2 & $2(2,1)$ & 7 & $10(10,3)$ & $-2,36$ & 0,01 \\
Neces. básicas/desejos & 15 & $21(21,9)$ & 9 & $11(11,3)$ & 1,96 & 0,04 \\
Família & 9 & $13(13,5)$ & 8 & $8(8,2)$ & 1,18 & 0,23 \\
Amizade & 5 & $5(5,2)$ & 9 & $9(9,3)$ & $-1,08$ & 0,27 \\
Não-Violência & 5 & $7(7,3)$ & 1 & $2(2,1)$ & 1,72 & 0,08 \\
Escola & 3 & $5(5,2)$ & 5 & $5(5,2)$ & 0,01 & 0,98 \\
\hline
\end{tabular}


Tabela 3. Frequências e porcentagens de respostas para o que é ser feliz, por faixa etária.

\begin{tabular}{lcccccccccc}
\hline & \multicolumn{3}{c}{$5-6$} & \multicolumn{2}{c}{$7-8$} & $9-10$ & \multicolumn{2}{c}{$11-12$} & \\
\cline { 2 - 11 } & $n$ & $f(\%)$ & $n$ & $f(\%)$ & $n$ & $f(\%)$ & $n$ & $f(\%)$ & $c^{2}$ & $p$ \\
\cline { 2 - 11 } Sentimentos & 21 & $24(37,5)$ & 17 & $22(26,2)$ & 22 & $29(22,5)$ & 20 & $29(27,6)$ & 4,92 & 0,18 \\
Self Positivo & 1 & $1(1,6)$ & 8 & $12(14,3)$ & 14 & $20(15,5)$ & 15 & $24(22,9)$ & 14,27 & 0,01 \\
Lazer & 14 & $18(28,1)$ & 12 & $15(17,9)$ & 6 & $10(7,8)$ & 6 & $9(8,6)$ & 18,78 & 0,01 \\
Neces. básicas/des. & 5 & $5(7,8)$ & 7 & $8(9,5)$ & 18 & $24(18,6)$ & 11 & $15(14,3)$ & 5,8 & 0,12 \\
Família & 0 & 0 & 7 & $9(10,7)$ & 12 & $16(12,4)$ & 8 & $9(8,6)$ & 8,56 & 0,03 \\
Amizade & 5 & $6(9,4)$ & 6 & $6(7,1)$ & 7 & $7(5,4)$ & 9 & $9(8,6)$ & 1,32 & 0,72 \\
Não-Violência & 4 & $5(7,8)$ & 6 & $6(7,1)$ & 6 & $8(6,2)$ & 1 & $1(1,6)$ & 5,6 & 0,13 \\
ESCOLA & 1 & $1(1,6)$ & 2 & $2(2,4)$ & 4 & $6(4,7)$ & 5 & $5(4,8)$ & 1,91 & 0,59 \\
\hline
\end{tabular}

Tabela 4. Frequência e porcentagem sobre como é uma pessoa feliz, por sexo.

\begin{tabular}{lcccccccc}
\hline & \multicolumn{2}{c}{ total } & \multicolumn{2}{c}{ Meninas } & Meninos \\
& $n$ & $f(\%)$ & $n$ & $f(\%)$ & $n$ & $f(\%)$ & $Z$ & $p$ \\
\hline & & 235 & & & & & $-2,41$ & 0,01 \\
CARACT. SUBJ. POSITIVAS & 127 & $(51,4)$ & 60 & $109(46)$ & 67 & $126(57,3)$ & & \\
Atividades & 60 & $60(21,2)$ & 33 & $56(23,6)$ & 27 & $41(18,6)$ & 1,30 & 0,19 \\
Neces. básicas/desejos & 40 & $58(12,7)$ & 23 & $34(14,3)$ & 17 & $24(10,9)$ & 1,10 & 0,27 \\
Caract. físicas & 44 & $51(11,1)$ & 24 & $30(12,7)$ & 20 & $21(9,5)$ & 1,05 & 0,29 \\
\hline
\end{tabular}

Como se pode notar na Tabela 1, aspectos positivos tomaram a maior parte das respostas: sentimentos positivos e descrições positivas do self. As análises mostraram que não houve diferenças significativas para sexo nas categorias sobre o que é ser feliz. A Tabela 2 mostra os resultados por tipo de escola. Foram constatadas duas diferenças significativas entre os tipos de escolas. As crianças que frequentavam escolas privadas apresentaram maior frequência na categoria "lazer", enquanto as crianças que frequentavam escolas públicas apresentaram maior frequência na categoria "satisfação de necessidades básicas e de desejos".

A Tabela 3 exibe as frequências e porcentagens de respostas para o que é ser feliz, por faixa etária.

Constataram-se três diferenças significativas entre as faixas etárias. Na categoria "self positivo" foram verificadas diferenças significativas entre as crianças da primeira faixa etária e as crianças da segunda $(p<0,05)$, da terceira $(p<0,01)$ e da quarta $(p<0,01)$ faixas etárias. Na categoria "lazer" a diferença significativa verificada foi localizada entre as crianças de 5-6 anos e as crianças de 9-10 anos ( $p<$ $0,01)$ e de 11-12 anos $(p<0,01)$. Diferenças foram constatadas na categoria "família" entre as crianças da primeira e da segunda faixa etária $(p<0,01)$, da terceira $(p<0,01)$ e da quarta $(p<0,05)$ faixas etárias.

A Tabela 4 mostra as frequências e porcentagens de respostas sobre a visão da criança entrevistada sobre como é uma pessoa feliz, por sexo.

Na questão 2, sobre "Como tu achas que uma pessoa feliz é?", as respostas das crianças possibilitaram a construção de seis categorias de unidades temáticas, similares às já citadas. As categorias com porcentagem inferior a 10 foram "outras respostas" e "não respondeu". Como se pode notar, mais da metade dos conteúdos identificados nas falas das crianças foram sobre características subjetivas positivas (nas tabelas, consta como "caract. subj. positivas"). O Teste Binomial para Diferenças entre Duas Proporções localizou 
Tabela 5. Frequência e porcentagem sobre como é uma pessoa feliz, por tipo de escola.

\begin{tabular}{lcccccc}
\hline & \multicolumn{2}{c}{ Pública } & \multicolumn{2}{c}{ Privada } \\
\cline { 2 - 7 } & $n$ & $f(\%)$ & $n$ & $f(\%)$ & $Z$ & $p$ \\
\hline CARACT. SUBJ. POSITIVAS & 36 & $80(64)$ & 32 & $71(57,3)$ & 1,08 & 0,27 \\
Atividades & 13 & $21(16,8)$ & 14 & $22(17,7)$ & $-0,19$ & 0,84 \\
Neces. básicas/desejos & 9 & $14(11,2)$ & 15 & $21(16,9)$ & 1,30 & 0,19 \\
Caract. físicas & 6 & $7(5,6)$ & 5 & $7(5,6)$ & $-0,01$ & 0,98 \\
\hline
\end{tabular}

Tabela 6. Frequência e porcentagem sobre como é uma pessoa feliz, por faixa etária.

\begin{tabular}{lcccccccccc}
\hline & \multicolumn{2}{c}{$5-6$} & \multicolumn{2}{c}{$7-8$} & \multicolumn{2}{c}{$9-10$} & \multicolumn{2}{c}{$11-12$} \\
\cline { 2 - 11 } & $n$ & $f(\%)$ & $n$ & $f(\%)$ & $n$ & $f(\%)$ & $n$ & $f(\%)$ & $c^{2}$ & $p$ \\
\hline CARACT. SUBJ. POSITIVAS & 16 & $18(25)$ & 28 & $45(46,9)$ & 46 & $95(59,7)$ & 35 & $73(58,4)$ & 27,7 & 0,00 \\
Atividades & 15 & $21(29,2)$ & 13 & $24(25)$ & 17 & $29(18,2)$ & 15 & $23(18,4)$ & 4,92 & 0,18 \\
Neces. básicas/desejos & 6 & $9(12,5)$ & 9 & $13(13,5)$ & 10 & $15(9,4)$ & 15 & $21(16,8)$ & 3,45 & 0,33 \\
Caract. físicas & 15 & $17(23,6)$ & 11 & $12(12,5)$ & 12 & $16(10,1)$ & 5 & $5(4)$ & 18,2 & 0,00 \\
\hline
\end{tabular}

uma diferença significativa. Como se pode acompanhar na Tabela 4, na categoria características subjetivas positivas os meninos citaram mais conteúdos do que as meninas.

A Tabela 5 apresenta as frequências e porcentagens sobre a visão da criança de como é uma pessoa feliz, por tipo de escola. Não foram encontradas diferenças significativas entre as respostas de crianças de escola pública e privada.

A Tabela 6 apresenta os resultados sobre como é uma pessoa feliz, por faixa etária. O Teste Qui-Quadrado localizou diferenças significativas na categoria "características subjetivas positivas" entre a primeira faixa etária e as demais $\left(1^{\mathrm{a}}\right.$ e $2^{\mathrm{a}} p<0,05 ; 1^{\mathrm{a}}$ e $3^{\mathrm{a}} p<0,01 ; 1^{\mathrm{a}}$ e $\left.4^{\mathrm{a}} p<0,01\right)$. Nas "características físicas", foram constatadas diferenças significativas entre as crianças de 5-6 anos e as crianças de 9-10 $(p<0,05)$ e 11-12 anos $(p<0,01)$.

Em resumo, os principais resultados encontrados no presente estudo foram:

- sentimentos positivos e self positivo foram os conteúdos mais escolhidos pelas crianças para descrever o que é ser feliz. Destaca-se que o lazer foi o terceiro conteúdo mais citado;

- as crianças de escola privada mencionaram mais o lazer como componente de ser feliz;

- as crianças de escola pública se destacaram ao definir que ser feliz é satisfazer necessidades básicas e de desejos;

- as crianças mais novas foram as que menos definiram que ser feliz envolve self positivo e família; todavia, essas crianças de 5-6 anos foram as que mais fizeram referência a lazer;

- na questão de como é uma pessoa feliz, mais da metade da amostra apontou características subjetivas positivas, com os meninos citando mais esses conteúdos, e as crianças menores pouco as mencionando;

- as crianças maiores pouco relataram características físicas de uma pessoa feliz.

\section{Discussão}

Este estudo buscou conhecer o que crianças de cinco a doze anos de idade, meninos e meninas de escolas públicas e privadas, entende por "ser feliz", bem como a forma que elas visualizam uma pessoa feliz. Dada a natureza combinada de pesquisa qualitativa e quantitativa, os dados coletados foram abundantes, e os resultados foram extensos. Assim, nessa oportunidade, serão discutidos os principais resultados (tanto os estatisticamente indicados como significativos, como aqueles que os autores elegeram como relevantes para o campo de estudos da felicidade e do bem-estar em crianças).

As crianças indicam que "ser feliz" é alcançado quando se tem um self positivo, isto é, quando se possui atitudes e traços positivos, entre eles ser altruísta, ético, empático, quando se possui capacidade para amar, para perdoar, 
quando se tem vocação, coragem, otimismo, entre outros. Pode-se observar isso nas seguintes verbalizações sobre o "ser feliz": "ajudar as pessoas", "ser legal", "acreditar no amor e na verdade", "perdoar o amigo", "ter opinião própria". Seligman e Csikszentmihalyi (2000), ao apresentarem a Psicologia Positiva e sua área de estudo, descrevem tais capacidades e traços em nível individual. A categoria "self positivo", descrita pelas crianças, fornece todos os indícios comprobatórios de que elas são capazes de identificar tais capacidades e traços positivos e sua importância para uma vida melhor. Ademais, a maioria das crianças descreveu uma pessoa feliz como alguém que possui características subjetivas positivas, e as crianças mais velhas foram as que menos citaram características físicas nessa descrição. Esses resultados são consistentes com achados de estudos sobre autoconceito infantil, como o de Shaffer (1989, 437-438).

Outra questão importante evocada pela descrição das crianças participantes são os caminhos tradicionais à felicidade: sentir-se bem (modelo hedônico), fazer o bem (modelo eudemônico) e o sentimento de flow (Siegel, 2011, p.10). O sentir-se bem aparece claramente nas respostas de sentimentos e emoções positivas, o fazer o bem está representado nas respostas de self positivo, ao passo que o flow está sugerido pelas crianças por meio das respostas sobre lazer, que incluem atividades que conduzem ao flow nas crianças, como o brincar e as atividades físicas e divertidas. A este respeito, Rocha e Souza (2011) discutem a relação estreita entre lazer e felicidade a partir dos estudos da psicologia positiva e do campo de estudos interdisciplinares do lazer.

Importante também foi notar que, além da ausência de diferenças de sexo na ideia de ser feliz como sentimentos e self positivo, também não se encontrou diferença de tipo de escola (pública ou privada). Considerou-se que a variável tipo de escola é o índice mais aproximado, obtido no presente estudo, para indicar nível socioeconômico das famílias das crianças. Assim, seria lícito considerar que, como indicado em pesquisas anteriores, a renda não é o principal indicador de felicidade, embora tenha seu papel. Este papel está sugerido no fato de que os conteúdos sobre lazer terem maior quantidade de respostas das crianças de escola privada.

A este respeito, Marcellino (1996), um dos pioneiros no estudo do lazer no Brasil, afirma que "o fator econômico é determinante desde a distribuição do tempo disponível entre as classes sociais, até as oportunidades de acesso à escola, e contribui para uma apropriação desigual do lazer. São as barreiras interclasses sociais" (p. 23). Outra questão importante foi a diferença significativa entre as crianças para a definição de ser feliz como a satisfação de necessidades básicas e de desejos. As crianças de escola pública citaram mais esta categoria, o que é outro indicativo de diferença socioeconômica nas oportunidades de vida em geral, e não somente relativas ao lazer.

Marcellino (1996) também propõe que há os fatores intraclasses sociais, como o sexo e a idade. Sobre o sexo, o autor discorre sobre a mulher e seu papel multifacetado como trabalhadora, doméstica, mãe, cidadã, etc. No entanto, na presente pesquisa esta distinção de sexo não se evidencia visto que a percepção da criança não demonstra conhecer tal diferença, ao menos nesta fase da vida. Já com respeito à idade, ele frisa que a criança, como não é considerada um ser produtivo, não se visualiza esta etapa da vida per se, mas como uma preparação para o futuro. Assim, muitas propostas de lazer para as crianças são bastante estruturadas, preparadas, com fins altamente educativos e com expectativas de performance. O puro e simples brincar, que possibilite o flow à criança, tem dado lugar a atividades mais preparatórias para a futura vida adulta.

Quanto às diferenças etárias na concepção de uma pessoa feliz, as crianças demonstraram o entendimento esperado para a idade, considerando-se as diferenças significativas identificadas. Tanto para a categoria lazer, como para a categoria self positivo, pode-se notar que o desenvolvimento cognitivo infantil evidencia-se nos resultados. À medida que as crianças vão referindo menos atividades físicas, divertidas e de brincar (lazer), vão adicionando, às suas idéias de como ser feliz, aqueles conteúdos relativos a boas ações e boas atitudes, como o ajudar e o compartilhar. Conforme o desenvolvimento infantil normal, é esperado o desinteresse pelo brincar com o passar dos anos. A habilidade de troca de papéis e de empatia refina-se dos cinco aos 12 anos, o que se detectou nas respostas das crianças. Esse resultado é condizente com os modelos teóricos tradicionais sobre o desenvolvimento sociocognitivo infantil, como Selman (1980) e Eisenberg (Koller \& Bernardes, 1997).

Para as crianças, a pessoa feliz é identificada por suas características subjetivas positivas, ou seja, sentimentos, humores, estados e traços positivos (mais de $50 \%$ das respostas das crianças). Outras descrições foram feitas baseadas nas atividades em que a pessoa feliz se envolve e realiza, na satisfação das necessidades básicas materiais e de desejo que ela possui e, por último, em suas características exteriores. Esses resultados remetem novamente ao papel das variáveis intrínsecas como determinantes do bem-estar subjetivo. Poder-se-ia identificar a categoria relativa aos aspectos subjetivos como os fatores intrínsecos relativos ao bem-estar subjetivo, as atividades realizadas pela pessoa feliz como os eventos de vida e a satisfação de necessidades básicas como os aspectos extrínsecos. Os índices das categorias obtidas podem estar refletindo os atuais modelos explicativos do bem-estar subjetivo, tendo em vista que a maior parte da variância do bem-estar está ligada aos aspectos intrínsecos do indivíduo. Portanto, para as crianças a pessoa feliz seria fruto de seus afetos, humores e emoções, assim como traços e estados, do relativo impacto de seus eventos de vida e de uma pequena parcela referente às suas necessidades básicas materiais atendidas. Segundo as crianças deste estudo, uma pessoa feliz possui um self positivo, realiza atividades de lazer, estuda, envolve-se em eventos de vida positivos, possui as suas necessidades básicas materiais e fisiológicas atendidas, assim como, suas necessidades de afeto e desejos.

Os estudos de Huebner e colaboradores (Dew \& Huebner, 1994; Huebner, 1991a, 1991b; Huebner \& Alderman, 1993; Terry \& Huebner, 1995) não indicam a existência 
de diferenças significativas nos níveis de satisfação de vida global de crianças e adolescentes quanto a algumas variáveis socioeconômicas. Entretanto, diante das limitações quanto à amostra analisada e dos apontamentos de autores que estudam tópicos específicos à infância, como Marcellino (1996) no caso do lazer, recomenda-se a realização de outros estudos que investiguem as possíveis diferenças existentes quanto aos domínios de satisfação de vida de crianças de realidades socioeconômicas distintas, uma vez que esses se demonstraram consistentes nessa amostra.

Também se pode considerar que à medida que as crianças entram na pré-adolescência, com consequente redução de investimento na família. Esse reflexo é observado nos percentuais de respostas na categoria "amizade" que, apesar de não ter apontado diferenças significativas, é verificado como uma tendência, já identificada pela psicologia do desenvolvimento (Souza, 2010).

\section{Considerações finais}

O presente estudo buscou conhecer a visão da criança sobre o que é ser feliz e de como é uma pessoa feliz. Os resultados trouxeram conhecimento interessante que agrega à escassa literatura nacional sobre o tema do bem-estar subjetivo na infância. Foram de destaque as diferenças de sexo, de faixa etária e de tipo de escola, a qual remete a considerações sobre distinções socioeconômicas entre as crianças.

Sobre o que é ser feliz, as crianças trouxeram mais conteúdos sobre sentimentos positivos e self positivo, com o lazer como o terceiro conteúdo mais citado. As crianças de escola privada mencionaram mais o lazer, e as de escola pública destacaram a satisfação de necessidades básicas e desejos. As crianças de 5-6 anos foram as que menos definiram que ser feliz envolve self positivo e família; todavia, essas mesmas crianças foram as que mais fizeram referência ao lazer.

$\mathrm{Na}$ questão de como é uma pessoa feliz, mais da metade da amostra (200 crianças) apontou características subjetivas positivas. Os meninos citaram mais esses conteúdos, e as crianças de 5-6 anos pouco os mencionaram. Além disso, as crianças de 11-12 anos pouco relataram características físicas de uma pessoa feliz.

As limitações do presente estudo acompanham as limitações da pesquisa maior que o envolve. Trata-se de uma amostra pequena, ainda que qualitativamente delineada para poder abarcar questões de diferenças de sexo, de tipo de escola e de faixa etária. O uso do tipo de escola como possível indicador de diferenças sócio-econômicas é reconhecido como uma limitação importante. Ademais, é uma amostra da área urbana de uma única cidade, Porto Alegre, o que deve ser levado em conta para a utilização destes resultados. Assim, novos insights podem ser alcançados através do presente trabalho, cujo desenlace pode ser importante para a população refletida nessa amostra. Estudos com amostra maior e mais abrangente, com outras regiões do Brasil, poderiam trazer dados interessantes acerca da percepção de como é uma pessoa feliz, bem como mais dados acerca das variáveis aqui tratadas, como o sexo e o tipo de escola.

Avaliar a felicidade, o bem-estar subjetivo e a satisfação de vida na criança requerem conhecer a forma como ela compreende o que significa uma pessoa feliz e de como uma pessoa feliz se apresenta aos outros. Isso é relevante porque as intervenções em satisfação de vida e bem-estar subjetivo também devem considerar domínios diferentes no exame dessa felicidade infantil. As orientações para uma criança altamente insatisfeita com sua família são orientações distintas daquelas a serem dadas a uma criança bastante infeliz com sua experiência escolar. Estas questões devem importar para o psicólogo clínico, para o psicólogo escolar, mas sobretudo para o psicólogo que compreenda que a busca pela felicidade não comporta apenas a supressão das experiências ruins, mas também do desenvolvimento e aprimoramento de habilidades, atitudes e comportamentos positivos.

\section{Referências}

Ayres, M., Ayres Jr., M., Ayres, D. L., \& Santos, A. S. (2000). BioEstat 2.0: aplicações estatísticas nas áreas das ciências biológicas e médicas. Belém: Soc. Civil Mamirauá/CNPq.

Bardin, L. (2011). Análise de conteúdo (L. Reto \& A. Pinheiro, Trads.). Lisboa: Edições 70.

Cia, F., \& Barham, E. J. (2009). Repertório de habilidades sociais, problemas de comportamento, autoconceito e desempenho acadêmico de crianças no início da escolarização. Estudos de Psicologia, 26(1), 45-55.

Dell'Aglio, D., Koller, S. H., \& Yunes, M. A. M. (Orgs.) (2006). Resiliência e psicologia positiva: interfaces do risco à proteção. São Paulo: Casa do Psicólogo.

Dew, T., \& Huebner, E. S. (1994). Adolescents' perceived quality of life: an exploratory investigation. Journal of School Psychology, 32, 185-199.

Freitas, L. B. de L., Silveira, P. G., \& Pietá, M. A. M. (2009). Sentimento de gratidão em crianças de 5 a 12 anos. Psicologia em Estudo, 14(2), 243-250.

Garcia, A., \& Pereira, P. (2008). Amizade na infância: um estudo empírico. Psic: revista de Psicologia da Vetor Editora, 9(1), 25-3.

Giacomoni, C. H., \& Hutz, C. S. (2008). Escala Multidimensional de Satisfação de Vida para Crianças: estudos de construção e validação. Estudos de Psicologia, 25(1), 23-35.

Harter, S. (1999). The construction of the self: a developmental perspective. New York: The Guilford Press.

Hinkle, P. E., Wiersma, W., \& Jurs, S. G. (1988). Applied statistics for the behavioral sciences (2a ed). Boston: Hougthon Miffin. 
Huebner, E. S. (1991a). Initial development of the student's life satisfaction scale. School Psychology International, 12, 229-238.

Huebner, E. S. (1991b). Correlates of life satisfaction in children. School Psychology_Quarterly, 6, 103-111.

Huebner, E. S., \& Alderman, G. L. (1993). Convergent and discriminant validation of a children's life satisfaction scale: Its relationship to self- and teacher-reported psychological problems and school functioning. Social Indicators Research, 30, 71-82.

Huebner, E. S. \& Gilman, R. (2002). An introduction to the multidimensional student's life satisfaction scale. Social Indicators Research, 60, 115-122.

Hutz, C. S. (Org.). (no prelo). Avaliação em psicologia positiva. São Paulo: Casa do Psicólogo.

Koller, S., \& Bernardes, N. (1997). Desenvolvimento moral pró-social: semelhanças e diferenças entre os modelos teóricos de Eisenberg e Kohlberg. Estudos de Psicologia, 2(2), 223-262.

La Taille, Y. (2006). A importância da generosidade no início da gênese da moralidade na criança. Psicologia: Reflexão e Crítica, 19(1), 9-17.

Levy, L. \& Jonathan, E. (2010). Minha família é legal? A família no imaginário infantil. Estudos de Psicologia, 27(1), 49-56.

Marcellino, N. C. (1996). Estudos do lazer: uma introdução. Campinas, SP: Autores Associados.
Rocha, M. A., \& Souza, L. K. de (2011). Um diálogo entre a psicologia positiva e os estudos do lazer. Em H. F. Isayama \& S. R. Da Silva (Orgs.), Estudos do lazer: um panorama (pp. 97-110). Rio de Janeiro: Apicuri.

Seligman, M. E. P., \& Csikszentmihalyi, M. (2000). Positive psychology: an introduction. American Psychologist, 55(1), 5-14.

Selman, R. L. (1980). The growth of interpersonal understanding. Orlando: Academic Press.

Shaffer, D. (1989). Developmental psychology: childhood and adolescence (2a ed.). Pacific Grove, CA: Brooks, Cole Publishing.

Siegel, R. D. (Org.). (2011). Positive Psychology: harnessing the power of happiness, mindfulness, and personal strenght. A Harvard Medical School Special Health Report. Boston, MA: Harvard Health Publications.

Souza, L. K. de. (2010). Avaliação da amizade na infância: da troca de papeis à melhor amizade. Em C. S. Hutz (Org.), Avanços em avaliação psicológica e neuropsicológica de crianças e adolescentes (pp. 7-29). São Paulo: Casa do Psicólogo.

Souza, L. K. de, Sperb, T. M., McCarthy, S., \& Biaggio, A. (2006). Brazilian children's conceptions of peace, war, and violence. Peace and Conflict: Journal of Peace Psychology, 12(1), 49-63.

Terry, T. \& Huebner, E. S. (1995). The relationship between selfconcept and life satisfaction in children. Social Indicators Research, $35,39-52$.

\section{Sobre os autores}

Claudia Hofheinz Giacomoni (giacomon@uol.com.br)

Universidade Federal do Rio Grande do Sul

Doutora em Psicologia

Luciana Karine de Souza (lucianak@fafich.ufmg.br)

Universidade Federal de Minas Gerais

Doutora em Psicologia

Claudio Simon Hutz (claudio.hutz@terra.com.br)

Universidade Federal do Rio Grande do Sul

Doutor em Psicologia

\section{Agradecimentos:}

L. Stein, R. Arendt, A. Biaggio, D. Bandeira, D. Dell'Aglio, S. Blatt, A. Tibulo, G. Mazzini, J. Zanchetin, A. Silveira, C. Oliveira, C. Hartmann, D. D'Incão, I. Gemeli, L. Azevedo e Souza, M. Fischborn, R. Ebert, T. Schmidt, V. Nachtigall, Z. Silveira. Apoio: CAPES; PDEE/CAPES; PPGPsicologia da Universidade Federal do Rio Grande do Sul. 